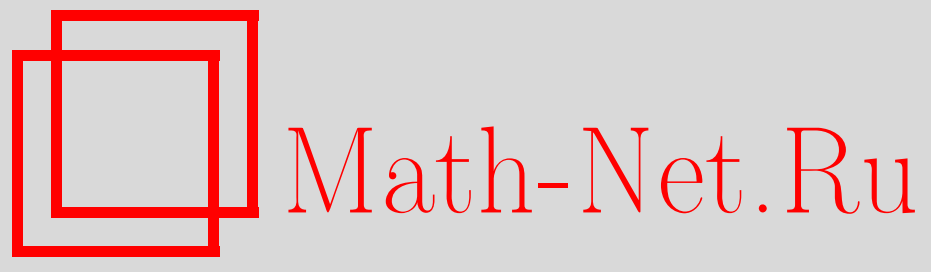

В. С. Монахов, Конечные группы с полунормальной холловой подгруппой, Матем. заметки, 2006, том 80, выпуск 4, 573-581

DOI: https://doi.org/10.4213/mzm2850

Использование Общероссийского математического портала Math-Net.Ru подразумевает, что вы прочитали и согласны с пользовательским соглашением http://www . mathnet.ru/rus/agreement

Параметры загрузки:

IP : 52.23 .180 .231

26 апреля 2023 г., 13:03:32

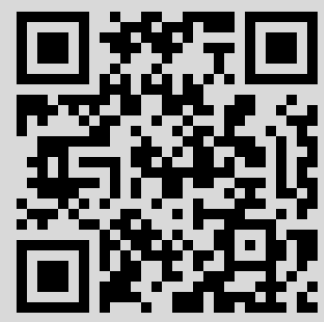




\section{КОНЕЧНЫЕ ГРУППЫ С ПОЛУНОРМАЛЬНОЙ ХолЛОВой ПоДГРУппой}

\section{В. С. Монахов}

Устанавливаются признаки $\pi$-разрешимости конечной группы с полунормальной $\pi$-холловой подгруппой и доказывается нильпотентность третьего коммутанта группы с полунормальными нециклическими силовскими подгруппами.

Библиография: 10 названий.

Все рассматриваемые группы предполагаются конечными. Подгруппа $H$ группы $G$ называется полунормалъной в $G$, если существует такая подгруппа $K$ из $G$, что $H K=G$ и $H K_{1}$ - собственная подгруппа группы $G$ для каждой подгруппы $K_{1}$ из $K$, отличной от $K$. В этой ситуации подгруппу $K$ назовем супердобавлением к подгруппе $H$ в группе $G$.

Очевидно, что каждая подгруппа простого индекса является полунормальной. Из леммы Кегеля [1] следует, что в простой группе полунормальная подгруппа всегда имеет простой индекс. Поэтому из [2] легко выводится полный список всех простых групп с полунормальной собственной подгруппой.

В непростых группах полунормальная подгруппа может иметь непростой индекс. Кроме того, всякая квазинормальная подгруппа (т.е. перестановочная со всеми подгруппами группы) является полунормальной. В симметрической группе $S_{4}$ и в полной линейной группе $G L(2,3)$ силовские 2-подгруппы полунормальны, но не квазинормальны. Отдельные свойства полунормальных подгрупп рассматривались в [3], [4]. В частности, в [3] замечена сверхразрешимость группы с полунормальными силовскими подгруппами.

В настоящей заметке изучаются группы с полунормальной холловой подгруппой. Доказываются следующие теоремы.

Теорема 1. Пусть в группе $G$ существует полунормальная $\pi$-холлова подгруппа $H$. Тогда группа $G$ т-разрешима в каждом из следующих случаев:

1) Н 2-нильпотентна;

2) Н разрешима и $3 \notin \pi$.

ТЕОРема 2. Пусть $G-\pi$-разрешимая группа с полунормальной $\pi$-холловой подгруппой $H$. Тогда

Работа выполнена при поддержке Белорусского республиканского фонда фундаментальных исследований, гранты № Ф04МС-060 и № Ф05-341.

(C) B. C. Монахов, 2006 
1) $H^{\prime} \subseteq O_{\pi}(G)$;

2) $l_{\pi}(G) \leqslant 2, l_{\pi^{\prime}}(G) \leqslant 2$

3) $l_{\pi}^{n}(G) \leqslant 1+n(H), l_{\pi}^{a}(G) \leqslant 1+d(H)$;

4) если $G_{\pi^{\prime}}$ q-сверхразрешима для некоторого $q \in \pi^{\prime}$, то группа $G$ q-сверхразрешима.

Теорема 3. Если в группе $G$ каждая нециклическая силовская подгруппа полунормальна, то группа $G$ разрешима и ее третий коммутант нильпотентен.

Напомним, что 2-нильпотентной называют группу с нормальной 2'-холловой подгруппой. Через $l_{\pi}(G), l_{\pi}^{n}(G), l_{\pi}^{a}(G)$ обозначается $\pi$-длина, нильпотентная $\pi$-длина и производная $\pi$-длина $\pi$-разрешимой группы $G$ соответственно; определения см. ниже. Как всегда, $H^{\prime}$ - коммутант группы $H$, а $O_{\pi}(G)$ - наибольшая нормальная $\pi$-подгруппа группы $G$; q-сверхразрешuмой группой называют группу, обладающую нормальным рядом, факторы которого либо $q^{\prime}$-группы, либо группы порядка $q$. Если $A$ и $B$ - подгруппы группы $G$, то $A^{B}=\left\langle A^{b} \mid b \in B\right\rangle$, в частности, $A^{G}$ - наименьшая нормальная подгруппа группы $G$, содержащая $A$.

Нам потребуются следующие леммы.

Лемма 1. 1. Если $H$ - полунормальная подгруппа группы $G u H \leqslant X \leqslant G$, mо $H$ полунормальна в $X$.

2. Если $H$ - полунормальная и $N$ - нормальная подгруппы группъ $G$, mо $H N / N$ полунормальная подгруппа в $G / N$.

3. Если $H$ - полунормальная подгруппа группь $G$ и $K$-ее супердобавление, то $H$ перестановочна с подгруппой $L^{g}$ для всех $L \leqslant K$ и всех $g \in G$. В частности, подгруппа $K^{g}$ будет супердобавлением $к$ подгруппе $H$ для каждого $g \in G$.

ДокАзАТЕЛЬСтво. Первые два свойства легко выводятся из определения полунормальной подгруппы. Проверим третье свойство. Пусть $g=a b, a \in K, b \in H$. Тогда $L^{a} \leqslant K$ и

$$
H L^{g}=H L^{a b}=\left(H L^{a}\right)^{b}=\left(L^{a} H\right)^{b}=L^{a b} H=L^{g} H .
$$

Так как $G=H K^{g}$, то $K^{g}$ - супердобавление к $H$ в $G$.

ЛЕмма 2. Если в группе $G$ существует полунормалъная $\pi$-холлова подгруппа $H$, то супердобавление кH в $G$ является $\pi^{\prime}$-холловой подгруппой группы $G$.

ДоказАтельство. Пусть $K$ - супердобавление к подгруппе $H$. Тогда $D=K \cap H$ является $\pi$-подгруппой. По свойству 3 из леммы 1 подгруппа $H$ перестановочна с $D^{g}$ для всех $g \in G$, поэтому $D^{G} \subseteq H$ и $D=K \cap H=K \cap D^{G}$ нормальна в $K$. Так как $|G: H|=|K: D|$, то $D-\pi$-холлова нормальная подгруппа в $K$. По теореме 1.18 .2 [5] существует в $K$ дополнение $K_{1}$ к подгруппе $D$, которое будет $\pi^{\prime}$-холловой подгруппой группы $G$. Ясно, что $G=H K_{1}$ и $K=K_{1}$ по определению супердобавления.

Лемма 3. Если $P$ и $Q$ - полунормалъные силовские $p$ - и q-подгруппъ группъ $G$, $p \neq q$, то $P Q-$ полунормальная $\{p, q\}$-холлова подгруппа в группе $G$.

ДокАзАтельство. Пусть $K$ - супердобавление к подгруппе $P$. По лемме 2 подгруппа $K$ будет $p^{\prime}$-холловой в группе $G$. Пусть $Q_{1}$ - силовская $q$-подгруппа из $K$. Так как $Q_{1}$ - силовская в $G$, то $Q_{1}^{x}=Q \leqslant K^{x}$ для некоторого $x \in G$. По свойству 3 
из леммы 1 подгруппа $P$ перестановочна с $Q$, т.е. $P Q$ является $\{p, q\}$-холловой подгруппой группы $G$ и $K^{x}$ будет супердобавлением к $P$. Поскольку $Q$ полунормальная в $K^{x}$, то супердобавление $K_{1}$ к $Q$ в $K^{x}$ будет $q^{\prime}$-холловой подгруппой в $K^{x}$, а значит, $\{p, q\}^{\prime}$-холловой подгруппой группы $G$. Если $K_{2}-$ произвольная подгруппа в $K_{1}$, то подгруппы $P$ и $Q$ перестановочны с $K_{2}$ и $G=(P Q) K_{1}$, т.е. $K_{1}$ - супердобавление к $P Q$ в $G$ и $P Q$ полунормальна в $G$.

Лемма 4. Пусть в группе $G$ существует полунормальная $\pi$-холлова подгруппа. Если $p>q$ для каждого $p \in \pi$ и каждого $q \in \pi^{\prime}$, то $G \pi$-замкнута.

ДоКАЗАтЕЛЬСтво. Воспользуемся индукцией по порядку группы $G$. Пусть $H-$ $\pi$-холлова подгруппа группы $G$ и $K$ - супердобавление к $H$ в $G$. По лемме 2 подгруппа $K$ является $\pi^{\prime}$-холловой в группе $G$. Предположим, что $M_{i}$ - максимальная подгруппа в $K$. Тогда $H M_{i}$ - подгруппа в группе $G$ и $H-\pi$-холлова подгруппа в $H M_{i}$. По индукции подгруппа $H$ нормальна в $H M_{i}$. Если $M_{1}$ и $M_{2}$ - две различные максимальные подгруппы в $K$, то $H$ нормальна в $\left\langle H M_{1}, H M_{2}\right\rangle=H K=G$ и лемма доказана. Если в $K$ только одна максимальная подгруппа, то $K$ - примарная циклическая подгруппа. Пусть $K-q$-подгруппа. По условию $p>q$ для каждого $p \in \pi(G), p \neq q$, т.е. $q$ - наименьший простой делитель порядка группы $G$. Теперь $H$ нормальна в $G$ по теореме 4.2 .8 [5].

Лемма 5. Если $H$ - полунормалъная подгруппа группъ $G$ и $K$ - ее супердобавление, то подгруппа $H^{L} \cap L^{H}$ субнормальна в $G$ для каждой подгруппь $L$ из $K$.

ДоКАЗАТЕЛЬСтво. По свойству 3 из леммы подгруппа $H$ перестановочна с $L^{g}$ для всех $L \leqslant K$ и всех $g \in G$. Теперь по теореме 7.2.5 [6] пересечение $H^{L} \cap L^{H}-$ субнормальная подгруппа группы $G$. Лемма доказана.

Группа $G$ называется $\pi$-отделимой, если каждый главный фактор $G$ является либо $\pi$-группой, либо $\pi^{\prime}$-группой; $\pi$-отделимая группа, у которой каждый $\pi$-главный фактор разрешим, называется $\pi$-разрешимой. В силу теоремы Томпсона-Фейта о разрешимости групп нечетного порядка каждая $\pi$-отделимая группа $\pi$-разрешима при $2 \in \pi$ и $\pi^{\prime}$-разрешима при $2 \in \pi^{\prime}$. Используемые в дальнейшем свойства $\pi$ отделимых и $\pi$-разрешимых групп можно найти в [5], [7; гл. 6, 9]. Дополнительно будут использоваться следующие понятия.

$\pi$-длина неединичной $\pi$-отделимой группы $G$ определяется как наименьшее число $\pi$-факторов во всех тех нормальных рядах группы $G$, в которых каждый фактор является либо $\pi^{\prime}$-группой, либо $\pi$-группой; $\pi$-длина $\pi$-отделимой группы $G$ обозначается через $l_{\pi}(G)$. Поскольку каждая $\pi$-отделимая группа является $\pi^{\prime}$-отделимой, то можно говорить о $\pi^{\prime}$-длине $l_{\pi^{\prime}}(G) \pi$-отделимой группы $G$.

Любая $\pi$-разрешимая группа обладает нормальным рядом, каждый фактор которого является либо $\pi^{\prime}$-группой, либо абелевой $\pi$-группой. Нильпотентная $\pi$-длина $l_{\pi}^{n}(G)$ неединичной $\pi$-разрешимой группы $G$ определяется как наименьшее число нильпотентных $\pi$-факторов во всех тех нормальных рядах группы $G$, в которых каждый фактор является либо $\pi^{\prime}$-группой, либо нильпотентной $\pi$-группой. Понятно, что при $\pi=\{p\}$ получаем обычное определение $p$-длины $p$-разрешимой группы (см. [5; п. 6.6]). Ясно также, что в этом случае $l_{\pi}(G)=l_{\pi}^{n}(G)=l_{p}(G)$. 
Производная $\pi$-длина $l_{\pi}^{a}(G)$ неединичной $\pi$-разрешимой группы $G$ определяется как наименьшее число абелевых $\pi$-факторов во всех тех нормальных рядах группы $G$, в которых каждый фактор является либо $\pi^{\prime}$-группой, либо абелевой $\pi$-группой.

Для единичной группы $E$ полагаем $l_{\pi}(E)=l_{\pi}^{n}(E)=l_{\pi}^{a}(E)=0$.

В случае, когда $\pi=\pi(G), \pi$-разрешимая группа $G$ становится разрешимой, нильпотентная $\pi$-длина $l_{\pi}^{n}(G)$ совпадет с нильпотентной длиной $n(G)$, а производная $\pi$-длина $l_{\pi}^{a}(G)$ - с производной длиной $d(G)$ группы $G$. При $\pi \subseteq \pi(G)$ и $\pi \neq \pi(G)$ понятия нильпотентной $\pi$-длины и производной $\pi$-длины также представляют самостоятельный интерес.

ЛЕмма 6 [7; лемма 9.1.3]. Если $G-\pi$-отделимая группа и $O_{\pi^{\prime}}(G)=E$, mо $C_{G}\left(O_{\pi}(G)\right) \subseteq O_{\pi}(G)$.

ЛЕмма 7. Если $\pi$-холлова подгруппа $\pi$-разрешимой группъ $G$ абелева, то $l_{\pi}^{a}(G)=l_{\pi}^{n}(G)=l_{\pi}(G) \leqslant 1$.

ДоказАтельство. Пусть вначале $O_{\pi^{\prime}}(G)=E$. По лемме $6 C_{G}\left(O_{\pi}(G)\right) \subseteq O_{\pi}(G)$. Так как $O_{\pi}(G) \subseteq G_{\pi}$ и $G_{\pi}$ абелева, то $G_{\pi} \subseteq C_{G}\left(O_{\pi}(G)\right)$ и $l_{\pi}^{a}(G) \leqslant 1$. Если $O_{\pi^{\prime}}(G) \neq$ $E$, то $l_{\pi}^{a}(G) / O_{\pi^{\prime}}(G) \leqslant 1$, а значит, и $l_{\pi}^{a}(G) \leqslant 1$. Поскольку $l_{\pi}(G) \leqslant l_{\pi}^{n}(G) \leqslant l_{\pi}^{a}(G)$, то лемма доказана.

ЛЕмма 8. Если $G-\pi$-отделимая группа, то $O_{\pi}(G / \Phi(G))=O_{\pi}(G) \Phi(G) / \Phi(G)$.

ДокАЗАТЕЛЬСтво. Пусть $K / \Phi(G)=O_{\pi}(G / \Phi(G))$ и $H-\pi$-холлова подгруппа из $K$. Тогда $K=H \Phi(G)$ и по лемме Фраттини

$$
G=K N_{G}(H)=H \Phi(G) N_{G}(H)=N_{G}(H),
$$

т.е. $H \subseteq O_{\pi}(G)$. Теперь

$$
O_{\pi}(G / \Phi(G)) \subseteq O_{\pi}(G) \Phi(G) / \Phi(G) .
$$

Обратное включение также выполняется, поэтому имеем равенство.

ЛЕмма 9 [8; следствие 7.7.1]. Если X-субнормальная $\pi$-разрешимая подгруппа группъ $G$, то $X^{G} \pi$-разрешима.

ЛЕмма 10. Если $\pi^{\prime}$-холлова подгруппа $\pi$-разрешимой группы $G$ является q-разрешимой, то и группа $G$ q-разрешима.

ДокАзАтЕЛьство. Пусть $N$ - минимальная нормальная подгруппа группы $G$. Факторгруппа $G / N$ удовлетворяет условию леммы, поэтому $G q$-разрешима. Значит, порядок $N$ должен делится на простое число $q$ и $N$ будет $\pi^{\prime}$-подгруппой. Теперь $N$ содержится в $\pi^{\prime}$-холловой подгруппе, а поскольку она $q$-разрешима и $N$ является прямым произведением простых изоморфных подгрупп, то $N$ становится $q$-подгруппой, а группа $G$-разрешимой.

ЛЕмма 11 [9; теорема 2]. Группа, являющаяся произведением 2-нильпотентной подгруппы и ииклической подгруппы, непроста. Если ииклический фактор имеет нечетный порядок, то группа разрешима.

ЛЕмма 12 [10]. Группа, являющаяся произведением разрешимых 3'-подгрупп, разрешима. 
ДОКАЗАТЕЛЬСТвО ТЕОРЕМЫ 1. Применим индукцию к порядку группы $G$. В силу свойств 1 и 2 из леммы 1 условия теоремы наследуются всеми факторгруппами группы $G$ и всеми подгруппами, содержащими подгруппу $H$. Поэтому можно считать, что в группе $G$ нет нетривиальных $\pi$-разрешимых нормальных подгрупп и в $G$ имеется единственная минимальная нормальная подгруппа; обозначим ее через $N$. По лемме 2 в $G$ существует $\pi^{\prime}$-холлова подгруппа $K$, которая является супердобавлением к подгруппе $H$ в группе $G$, т.е. $H K_{1}$ - собственная подгруппа в $G$ для любой собственной подгруппы $K_{1}$ из $K$. По свойству 1 из леммы 1 подгруппа $H$ полунормальна в $H K_{1}$ и $H^{H K_{1}}=H^{K_{1}} \subseteq H K_{1} \neq G$, поэтому $H K_{1}-\pi$-разрешимая подгруппа по индукции. По свойству 3 из леммы 1 подгруппа $H$ перестановочна с подгруппой $K_{1}^{x}$ для каждого $x \in G$. По лемме 5 пересечение $D=H^{K_{1}} \cap K_{1}^{H}$ является субнормальной подгруппой группы $G$. Подгруппа $D \pi$-разрешима, поскольку $D \subseteq H^{K_{1}}$. По лемме 9 подгруппа $D^{G}$ является $\pi$-разрешимой и нормальной в $G$, поэтому $E=D=H^{K_{1}} \cap K_{1}^{H}$ и

$$
\left[H, K_{1}\right] \subseteq\left[H^{K_{1}}, K_{1}^{H}\right] \subseteq H^{K_{1}} \cap K_{1}^{H}=E .
$$

Значит, подгруппа $H$ централизует каждую собственную подгруппу из $K$. Если $K$ содержит две различные максимальные подгруппы, то $H$ централизует подгруппу $K$, поэтому $H$ нормальна в $G$. Следовательно, в группе $K$ только одна максимальная подгруппа, поэтому $K$ - циклическая $p$-группа для некоторого простого $p$.

Если $|K|>p$, то максимальная подгруппа из $K$ будет неединичной нормальной подгруппой группы $G$, противоречие. Значит, $|K|=p$. Ясно, что $p>3$. Теперь группа $G$ разрешима по лемме 11 или по лемме 12 .

ПримеР 1. В знакопеременной группе $A_{5}$ подгруппа $A_{4}$ является $\{2,3\}$-холловой полунормальной подгруппой. Значит, группа с 2-замкнутой, а тем более просто с разрешимой $\pi$-холловой подгруппой может быть не $\pi$-разрешимой.

СлЕДСтвиЕ 1. Если в группе $G$ существует полунормальная $\pi$-холлова подгруппа нечетного порядка, то $G$ т-разрешима.

СледСтвиЕ 2. Если в группе $G$ существует полунормалъная 2-нильпотентная холлова подгруппа нечетного индекса, то $G$ разрешима.

СлЕДСТвиЕ 3. Если в группе $G$ силовская p-подгруппа полунормальна, то $G$ pразрешима.

ДокАЗАТЕЛЬСтво тЕОРЕмы 2. Докажем утверждение 1). Применим индукцию к порядку группы $G$. Предположим, что $N=O_{\pi}(G) \neq E$. Тогда $G / N-\pi$ разрешимая группа с полунормальной $\pi$-холловой подгруппой $H / N$. По индукции $(H / N)^{\prime} \subseteq O_{\pi}(G / N)=E$. Поскольку $(H / N)^{\prime}=H^{\prime} N / N$, то $H^{\prime} N=N$ и $H^{\prime} \subseteq N$.

Теперь будем считать, что $O_{\pi}(G)=E$, и покажем, что подгруппа $H$ абелева. Предположим, что подгруппа Фраттини $\Phi(G)$ группы $G$ неединична. Тогда по индукции

$$
(H \Phi(G) / \Phi(G))^{\prime} \subseteq O_{\pi}(G / \Phi(G)) .
$$

По лемме $8 O_{\pi}(G / \Phi(G))=E$ и $H \Phi(G) / \Phi(G)$ абелева. Но теперь

$$
H^{\prime} \subseteq(H \Phi(G))^{\prime} \subseteq \Phi(G),
$$


а так как $\Phi(G)-\pi^{\prime}$-группа, то $H^{\prime}=E$ и $H$ абелева. Следовательно, можно считать, что $\Phi(G)=E$.

Предположим, что $B=O_{\pi^{\prime}}(G) H$ - собственная подгруппа группы $G$. Тогда $H^{\prime} \subseteq O_{\pi}(B)$ по индукции. Но

$$
O_{\pi}(B) \subseteq C_{G}\left(O_{\pi^{\prime}}(G)\right) \subseteq O_{\pi^{\prime}}(G)
$$

по лемме 6. Значит, $O_{\pi}(B)=E$ и $H$ абелева. Итак, группа $G=O_{\pi^{\prime}}(G) H$.

Пусть $M_{i}$ - максимальная подгруппа в $O_{\pi^{\prime}}(G)$. Так как $H$ полунормальна в $G$, то $H M_{i}$ - собственная подгруппа в группе $G$. Кроме того,

$$
M_{i} H \cap O_{\pi^{\prime}}(G)=M_{i}\left(H \cap O_{\pi^{\prime}}(G)\right)=M_{i}
$$

и $M_{i}$ нормальна в $M_{i} H$. По индукции,

$$
H^{\prime} \subseteq O_{\pi}\left(H M_{i}\right) \subseteq C_{H M_{i}}\left(M_{i}\right)
$$

Если в группе $O_{\pi^{\prime}}(G)$ имеются две различные максимальные подгруппы $M_{1}$ и $M_{2}$, то

$$
H^{\prime} \subseteq C_{G}\left(M_{1}\right) \cap C_{G}\left(M_{2}\right) \subseteq C_{G}\left(\left\langle M_{1}, M_{2}\right\rangle\right) \subseteq C_{G}\left(O_{\pi^{\prime}}(G)\right) \subseteq O_{\pi^{\prime}}(G) .
$$

Значит, $H^{\prime}=E$ и $H$ абелева. Если в группе $O_{\pi^{\prime}}(G)$ только одна максимальная подгруппа, то $O_{\pi^{\prime}}(G)$ является циклической примарной подгруппой. Но группа автоморфизмов циклической группы абелева. Поэтому $G / C_{G}\left(O_{\pi^{\prime}}(G)\right)$ абелева. Отсюда следует, что $H$ абелева. Первое утверждение теоремы 2 доказано.

Докажем пункты 2) и 3$)$. Так как $\pi$-холлова подгруппа в группе $G / O_{\pi}(G)$ абелева, то

$$
l_{\pi}\left(G / O_{\pi}(G)\right)=l_{\pi}^{n}\left(G / O_{\pi}(G)\right)=l_{\pi}^{a}\left(G / O_{\pi}(G)\right)=1
$$

по лемме 7 . Но $n\left(O_{\pi}(G)\right) \leqslant n(H), d\left(O_{\pi}(G)\right) \leqslant d(H)$, поэтому

$$
l_{\pi}(G) \leqslant 2, \quad l_{\pi}^{n}(G) \leqslant 1+n(H), \quad l_{\pi}^{a}(G) \leqslant 1+d(H) .
$$

Для получения оценки $l_{\pi^{\prime}}(G) \leqslant 2$ применим индукцию к порядку группы $G$. По индукции можно считать, что $O_{\pi}(G)=E$, поэтому $\pi$-холлова подгруппа в $G$ абелева и $l_{\pi}(G) \leqslant 1$ по лемме 7 . Но теперь $l_{\pi^{\prime}}(G) \leqslant 2$.

Докажем 4). Воспользуемся индукцией по порядку группы $G$. По лемме 2 в $G$ существует $\pi^{\prime}$-холлова подгруппа $K$ и $H$ перестановочна со всеми подгруппами из $K$. Условия наследуются всеми факторгруппами. Если $\Phi(G) \neq E$, то по индукции факторгруппа $G / \Phi(G) \quad q$-сверхразрешима, теперь $G \quad q$-сверхразрешима по следствию 4.2 .2 [8]. Ясно, что $O_{q^{\prime}}(G)=O_{\pi}(G)=E$ и в группе $G$ единственная минимальная нормальная подгруппа $N$, причем $N$ является $\pi^{\prime}$-подгруппой. Поэтому $N \subseteq K$. Пусть $L$ - минимальная нормальная подгруппа в $K$, содержащаяся в $N$. Поскольку подгруппа $K \quad q$-сверхразрешима, то $L$ является либо $q^{\prime}$-подгруппой, либо $L$ имеет порядок $q$. Подгруппа $L$ субнормальна в $G$ и $\pi\left(L^{G}\right)=\pi(L)$ по следствию 7.7.2 [8]. Так как $O_{q^{\prime}}(G)=E$, то $|L|=q$. Из полунормальности подгруппы $H$ следует, что произведение $H L$ является подгруппой группы $G$ и $H L \cap N=L(H \cap N)=L-$ нормальная подгруппа в $H L$, а значит, и в $G$. Поэтому $L=N$ и факторгруппа $G / C_{G}(N)$ циклическая как группа автоморфизмов группы $N$ простого порядка $q$. 
По теореме 3.4.5 [5] при $\Phi(G)=E$ подгруппа Фиттинга $F(G)$ является прямым произведением абелевых минимальных нормальных подгрупп группы $G$, поэтому

$$
F(G)=F\left(O_{\pi^{\prime}}(G)\right)=N=O_{q}(G) .
$$

По лемме 10 группа $G$-разрешима, а по лемме $6 N=C_{G}(N)$ и группа $G$ становится метациклической, а значит, и сверхразрешимой. Теорема доказана.

ПримеР 2. Полная линейная группа $G L(2,3)$ имеет ряд коммутантов

$$
G L(2,3)>S L(2,3)>Q>I>E,
$$

где $Q=(G L(2,3))^{\prime \prime}$ - группа кватернионов порядка $8, I=Z(G L(2,3))=G L(2,3)^{\prime \prime \prime}$ центр группы $G L(2,3),|I|=2$. Поэтому

$$
l_{2}(G L(2,3))=l_{2}^{n}(G L(2,3))=2, \quad l_{2}^{a}(G L(2,3))=3 .
$$

В $G L(2,3)$ силовская 2-подгруппа имеет простой индекс, поэтому она полунормальна. Значит, оценки длин в пунктах 2), 3) теоремы 2 являются точными.

СлеДСТВИе 4. Пусть $p$ - простое число, $P$ - силовская $p$-подгруппа группь $G$ u $\pi=\pi(G) \backslash\{p\}$. Если подгруппа $P$ полунормальна в $G$, то справедливы следующие утверждения:

1) группа $G$ р-разрешима и $P^{\prime} \leqslant O_{p}(G)$;

2) $l_{p}(G) \leqslant 2, l_{\pi}(G) \leqslant 2$ u $l_{p}^{a}(G) \leqslant 1+d(P)$;

3) если $p^{\prime}$-холлова подгруппа группы $G$ q-сверхразрешима для некоторого $q \in \pi$, то группа $G$ q-сверхразрешима.

СлеДСТВИЕ 5. Бипримарная $\{p, q\}$-группа $G$ с полунормальной силовской $p$-подгруппой является q-сверхразрешимой группой, $u n(G) \leqslant 3$. Кроме того, $G$ p-замкнута при $р>q$.

ДокАЗАтЕЛЬСтво. $q$-сверхразрешимость группы $G$ вытекает из третьего утверждения следствия 4. Если $q<p$, то группа $G$-замкнута по лемме 4.

ПримеР 3. Симметрическая группа $S_{4}$ имеет нильпотентную длину 3 , и в ней силовская 2-подгруппа полунормальна. Поэтому оценка нильпотентной длины группы в следствии 5 точная.

СлЕДСТвИЕ 6. Пусть $G$ - группа $u r \in \pi(G)$. Если в группе $G$ силовские $p$ подгруппы полунормальны для всех $p \in \pi(G) \backslash\{r\}$, то группа $G$ разрешима и $r$ сверхразрешима. В частности, если в группе $G$ все силовские подгруппы полунормальны, то $G$ сверхразрешима.

ДокАЗАтельство. Пусть $\pi=\pi(G) \backslash\{r\}$. По теореме 2 группа $G$ p-разрешима для всех $p \in \pi$, поэтому группа $G \pi$-разрешима, а так как $|\pi(G) \backslash \pi|=|\{r\}|=1$, то группа $G$ разрешима и $r$-сверхразрешима. Если в группе $G$ все силовские подгруппы полунормальны, то $G$-сверхразрешима для каждого $p \in \pi(G)$, поэтому группа $G$ сверхразрешима.

Отметим, что в [3] также доказана сверхразрешимость группы с полунормальными силовскими подгруппами. Но в [3] использовалась классификация простых групп. Наше доказательство классификацию простых групп не использует. 
СлЕДСТвИЕ 7. Пусть $G$ - группа $u r, s \in \pi(G)$. Если в группе $G$ силовские $p$ подгруппы полунормальны для всех $p \in \pi(G) \backslash\{r, s\}$, то группа $G$ разрешима.

ДокАзАтельство. Пусть $\pi=\pi(G) \backslash\{r, s\}$. По теореме 2 группа $G$ p-разрешима для всех $p \in \pi$, поэтому группа $G \pi$-разрешима. Теперь группа $G$ обладает нормальным рядом, факторы которого либо разрешимые $\pi$-группы, либо $\{r, s\}$-группы. Так как бипримарные группы разрешимы, то группа $G$ разрешима.

ДоКАЗАТЕЛЬСТво тЕОРЕмы 3. Пусть $\pi(G)=\pi \cup \tau$, причем для каждого $p \in \pi$ силовская $p$-подгруппа полунормальна, а для каждого $q \in \tau$ силовская $q$-подгруппа циклическая. Если $\pi=\varnothing$ или $\tau=\varnothing$, то $G$ сверхразрешима либо по теореме 4.2.11 [5], либо по следствию 6. В этом случае по теореме 6.9.1 [5] коммутант группы $G$ нильпотентен и теорема 3 доказана. Поэтому будем считать, что $\pi \neq \varnothing \neq \tau$.

По теореме 2 группа $G$ p-разрешима для всех $p \in \pi$, поэтому группа $G \pi$ разрешима. Теперь группа $G$ обладает нормальным рядом, факторы которого либо разрешимые $\pi$-группы, либо $\tau$-группы. Так как в $\tau$-группе все силовские подгруппы циклические, то по теореме 4.2.11 [5] все $\tau$-факторы этого ряда метациклические. Следовательно, группа $G$ разрешима. Теперь группа $G=A B$, где $A-\pi$-холлова подгруппа, а $B-\tau$-холлова подгруппа. По лемме $3 A$ - полунормальная $\pi$-холлова подгруппа группы $G$ и $A^{\prime} \subseteq O_{\pi}(G)$ по теореме 2 .

Пусть $N$ - неединичная нормальная подгруппа группы $G$. Ясно, что в $G / N$ силовские подгруппы либо полунормальны, либо циклические. По индукции подгруппа

$$
(G / N)^{\prime \prime \prime}=G^{\prime \prime \prime} N / N \simeq G^{\prime \prime \prime} / G^{\prime \prime \prime} \cap N
$$

нильпотентна, где $G^{\prime \prime \prime}$ - третий коммутант группы $G$. Если в $G$ существуют две минимальные нормальные подгруппы $N_{1}$ и $N_{2}$, то $N_{1} \cap N_{2}=E$ и $G$ изоморфна подгруппе из прямого произведения $\left(G / N_{1}\right) \times\left(G / N_{2}\right)$. Так как коммутант прямого произведения совпадает с прямым произведением коммутантов сомножителей, то подгруппа $G^{\prime \prime \prime}$ будет содержаться в произведении третьих коммутантов групп $G / N_{1}$ и $G / N_{2}$, а значит, будет нильпотентной. Если $\Phi(G) \neq E$, то $G^{\prime \prime \prime} / G^{\prime \prime \prime} \cap \Phi(G)$ нильпотентна, а по следствию 4.2 .1 [8] подгруппа $G^{\prime \prime \prime}$ нильпотентна. Таким образом, следует считать, что $\Phi(G)=E$ и в $G$ единственная минимальная нормальная подгруппа. Но в этом случае подгруппа Фиттинга $F(G)$ является минимальной нормальной подгруппой, $F(G)=C_{G}(F(G))$ и $F(G)=O_{r}(G)$ для некоторого простого $r$; см. [5; теоремы 3.4.2, 3.4.5].

Если $r \in \tau$, то $F(G) \subseteq B$ и $F(G)$ циклическая. Теперь $|F(G)|=r$ и факторгруппа $G / F(G)$ - циклическая группа порядка, делящего $r-1$. Поэтому второй коммутант $G^{\prime \prime}$ нильпотентен.

Пусть $r \in \pi$, тогда $F(G) \subseteq A$. Так как $A$ сверхразрешима по следствию 6 , то $A$ дисперсивна и ее коммутант $A^{\prime}$ нильпотентен, см. [5; теорема 6.9.1]. Равенство $F(G)=C_{G}(F(G))$ вынуждает подгруппу $A^{\prime}$ быть $r$-подгруппой, а $r$ - наибольшим простым числом в $\pi$. Поскольку $A^{\prime} \subseteq O_{\pi}(G) \subseteq A$, то $O_{\pi}(G)$ дисперсивна, поэтому $A^{\prime} \subseteq O_{r}\left(O_{\pi}(G)\right) \subseteq F(G)$. Теперь $A / F(G)$ - абелева $\pi$-холлова подгруппа группы $G / F(G)$.

По теореме 4.2.11 [5] подгруппа $B=B^{\prime} B_{1}$, где $B^{\prime}$ и $B_{1}$ - циклические холловы подгруппы. Подгруппа $A$ перестановочна с подгруппами $B^{\prime}$ и $B_{1}$, поскольку $A$ 
полунормальна. Теперь факторгруппа

$$
G / F(G)=(A / F(G))\left(B^{\prime} F(G) / F(G)\right)\left(B_{1} F(G) / F(G)\right)
$$

является произведением абелевых попарно перестановочных подгрупп взаимно простых порядков. По теореме 6.14.16 [5] производная длина факторгруппы $G / F(G)$ не превышает 3. Это означает, что третий коммутант группы $G$ нильпотентен. Теорема 3 доказана.

\section{СПИСОК ЦИТИРОВАННОЙ ЛИТЕРАТУРЫ}

[1] O. Kegel, "Produkte nilpotenter Gruppen", Arch. Math., 12 (1961), 90-93.

[2] R. M. Guralnick, "Subgroups of prime power index in a simple group", J. Algebra, 81:2 (1983), 304-311.

[3] В.В. Подгорная, "Полунормальные подгруппы и сверхразрешимость конечных групп”, Весці НАН Беларусі. Сер. фіз.-матэм. навук, 2000, № 4, 22-25.

[4] Su Xiongying, "On semi-normal subgroups of finite group", J. Math. (Wuhan), 8(1) (1988), 7-9.

[5] B. Huppert, Endliche Gruppen, I, Springer-Verlag, Berlin-Heidelberg-New York, 1967.

[6] J. C. Lennox, S. E. Stonehewer, Subnormal Subgroups of Groups, Clarendon Press, Oxford, 1987.

[7] B. Huppert, N. Blackburn, Finite Groups, II, Springer-Verlag, Berlin-Heidelberg-New York, 1982.

[8] Л. А. Шеметков, Формации конечных групп, Наука, М., 1978.

[9] В. С. Монахов, "Произведение разрешимой и циклической групп", VI Всесоюзный симпозиум по теории групn, Сб. научных трудов, Наукова думка, Киев, 1980, 188195.

[10] С. А. Сыскин, "Об одном вопросе Р. Бэра", Сиб. матем. ж., 20:3 (1979), 679-681.

В. С. Монахов

Поступило

Гомельский университет им. Ф. Скорины, Беларусь

08.12 .2005

E-mail: monakhov@gsu. unibel.by 